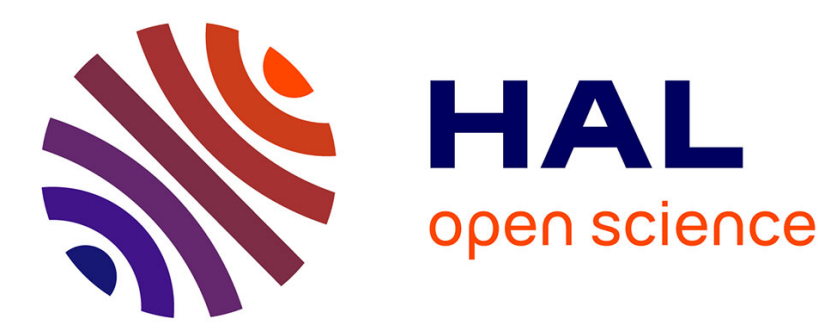

\title{
A two-dimensional relaxation scheme for the hybrid modelling of two-phase flows
}

Kateryna Dorogan, Jean-Marc Hérard, Jean-Pierre Minier

\section{To cite this version:}

Kateryna Dorogan, Jean-Marc Hérard, Jean-Pierre Minier. A two-dimensional relaxation scheme for the hybrid modelling of two-phase flows. Finite Volumes for Complex Applications VI, Jun 2011, Prague, Czech Republic. pp.351-359, 10.1007/978-3-642-20671-9_37 . hal-01580269

\section{HAL Id: hal-01580269 \\ https://hal.science/hal-01580269}

Submitted on 5 Dec 2019

HAL is a multi-disciplinary open access archive for the deposit and dissemination of scientific research documents, whether they are published or not. The documents may come from teaching and research institutions in France or abroad, or from public or private research centers.
L'archive ouverte pluridisciplinaire HAL, est destinée au dépôt et à la diffusion de documents scientifiques de niveau recherche, publiés ou non, émanant des établissements d'enseignement et de recherche français ou étrangers, des laboratoires publics ou privés. 


\title{
A two-dimensional relaxation scheme for the hybrid modelling of two-phase flows
}

\author{
Kateryna Dorogan, Jean-Marc Hérard and Jean-Pierre Minier
}

\begin{abstract}
Recently, a new relaxation scheme for hybrid modelling of two-phase flows has been proposed. This one allows to obtain stable unsteady approximations for a system of partial differential equations containing non-smooth data. This paper is concerned with a two-dimensional extension of the present method, in which two alternative relaxation schemes are compared. A partial analysis of continuous stability is given.
\end{abstract}

\section{Introduction}

This paper deals with the modelling and the numerical simulation of polydispersed turbulent two-phase flows, where one phase is a turbulent fluid (considered to be a continuum) and the other appears as separate inclusions carried by the fluid (solid particles, droplets or bubbles). Such a kind of flows can be encountered in many industrial situations (combustion, water sprays, smokes) and in some environmental problems. Despite the need of their accurate prediction, the physical complexity of these processes is so broad that existing methods are either too expensive (in calculation cost) or not sufficiently accurate. A hybrid approach recently proposed in [5] enables to reach an acceptable compromise between the physical realism and a cheap numerical treatment. A similar approach has been addressed in [13] for a single-phase turbulent flow. For two-phase flows, it consists in coupling two classic approaches (Eulerian and Lagrangian) in the particle phase description, while for the fluid phase only the Eulerian description is used. Given the consistency of the fluid and the particle descriptions, this method allows to gather the advantages of classic approaches: high level of physical description, lower calculation costs,

Kateryna Dorogan: EDF R\&D, MFEE, 6 quai Watier, F-78400 Chatou, and: LATP, CMI, 39 rue Joliot Curie, F-13453 Marseille (kateryna.dorogan@edf.fr)

Jean-Marc Hérard: EDF R\&D, MFEE, 6 quai Watier, F-78400 Chatou (jean-marc.herard@edf.fr) Jean-Pierre Minier: EDF R\&D, MFEE, 6 quai Watier, F-78400 Chatou (jean-pierre.minier@edf.fr) 
correct treatment of non-linearities and polydispersity, expected values free from statistical error. The Lagrangian part of the particle phase description is given by the stochastic differential equations:

$$
\begin{aligned}
& d x_{p, i}=U_{p, i} d t \\
& d U_{p, i}=\frac{1}{\tau_{p}}\left(U_{s, i}-U_{p, i}\right) d t+g_{i} d t \\
& d U_{s, i}=A_{i}\left(t, Z_{t}, p(t ; z), Y_{t}\right) d t+B_{i}\left(t, Z_{t}, p(t ; z), Y_{t}\right) d W_{t, i},
\end{aligned}
$$

where $p(t ; z)$ stands for the probability density function (pdf) of the particle state vector $Z_{t}=\left(x_{p}, U_{p}, U_{s}\right)$ with $x_{p}(t)$ the particle position, $U_{p}\left(x_{p}(t), t\right)$ the particle velocity, $U_{f}\left(x_{f}(t), t\right)$ the fluid velocity, $U_{s}\left(x_{p}(t), t\right)$ the fluid velocity seen at the particle position and the local relative velocity $U_{r}=U_{s}-U_{p} . Y_{t}$ represents external mean fields, i.e. the fluid mean fields defined at particle locations [12]. Then, using corresponding Fokker-Planck equation we deduce from (1) a system of partial differential equations for the mean particle concentration and the mean particle flow rate $\left(\alpha_{p}, \alpha_{p}\left\langle U_{p, i}^{E}\right\rangle\right)$, which represents an Eulerian description of the particle phase:

$$
\begin{aligned}
& \partial_{t} \alpha_{p}^{E}+\partial_{x_{i}}\left(\alpha_{p}^{E}\left\langle U_{p, i}^{E}\right\rangle\right)=0 \\
& \partial_{t}\left(\alpha_{p}^{E}\left\langle U_{p, i}^{E}\right\rangle\right)+\partial_{x_{j}}\left(\alpha_{p}^{E}\left(\left\langle U_{p, i}^{E}\right\rangle\left\langle U_{p, j}^{E}\right\rangle+\left\langle u_{p, i} u_{p, j}\right\rangle^{L}\right)\right)=\alpha_{p}^{E} g_{i}+\left\langle\alpha_{p}^{E} U_{r, i}^{L} / \tau_{p}^{L}\right\rangle
\end{aligned}
$$

Usually, only one among the two systems (1), (2) is solved. However, in this case we are faced with shortcomings of the standard methods. In fact, system (1) contains a bias-error and thus needs calculations with a larger number of particles, whereas the Reynolds stress term $\left\langle u_{p, i} u_{p, j}\right\rangle^{L}$ in system (2) is not closed. The new hybrid approach consists in solving both of these systems at the same time. Thus, the terms with superscript " $L$ ", calculated with a better accuracy in the Lagrangian part of the model, are provided to the Eulerian part (2). The latter, in turn, gives the values of $\left\langle U_{p, i}^{E}\right\rangle$ free from statistical error, that enable computations with a smaller number of particles in (1). Hence, for the same accuracy, the total calculation cost is reduced with reference to the Lagrangian approach. Here, in order to guarantee the coupling of descriptions (1)-(2), we have to choose the averaging and interpolation procedures. Moreover, such a coupling introduces noisy quantities (computed by the stochastic equations) in the Eulerian part of the model, which presents an important convective part and thus requires a stabilization.

A specific relaxation approach was proposed in $[6,7]$ to tackle this problem in a one-dimensional case. It relies both on upwinding techniques and relaxation tools [11] and allows to obtain stable unsteady approximations of solutions of system (2). Actually, two alternative relaxation systems were compared and some analysis of their stability was given.

The present paper is concerned with a two-dimensional extension of the relaxation approach. In section 1, we still propose two forms of the relaxation system that 
are very similar but that need a different numerical treatment. We will give some results on the stability in section 3 and briefly describe the numerical treatment in section 4. At last, a few numerical results will be given.

\section{Relaxation approach in a two-dimensional framework}

In order to compute stable approximations of the solution $\underline{W}^{t}=\left(\alpha_{p}^{E}, \alpha_{p}^{E}\left\langle U_{p, i}^{E}\right\rangle\right)$, $i=1,2$ of system (2), we proceed as follows. Since non-smooth external data are introduced in the system, we are formally interested in finding discontinuous solutions. However, for system (2) the Riemann problem can not be defined in an usual way, because the term $\underline{R}^{L}=\left\langle u_{p} u_{p}\right\rangle^{L}$ is not closed as $\underline{\underline{R}}^{L}(W)$. To overcome this difficulty, a relaxation technique was proposed in $[8,9]$, which is grounded on ideas developed in [3]. It consists in introducing supplementary equations that govern the time evolution of the Reynolds stresses, so that the relaxation system is hyperbolic and preserves the realizability of solutions.

From now on, we omit the superscripts " $E$ " and we denote by $\rho=\alpha_{p} \rho_{p}$ the mean density distribution of the particles in the domain, by $U_{i}=\left\langle U_{p, i}\right\rangle, i=1,2$ the mean particle velocity and by $R_{i j}=\left\langle u_{p, i} u_{p, j}\right\rangle,(i, j) \in\{1,2\}^{2}$ the "Reynolds stress" tensor. We propose the following natural expression for an extended system corresponding to (2):

$$
\begin{aligned}
& \partial_{t} \rho+\partial_{x_{j}}\left(\rho U_{j}\right)=0 \\
& \partial_{t}\left(\rho U_{i}\right)+\partial_{x_{j}}\left(\rho U_{i} U_{j}\right)+\partial_{x_{j}}\left(\rho R_{i j}^{L}\right)=\rho g_{i}+\rho\left\langle U_{r, i} / \tau_{p}\right\rangle^{L} \\
& \partial_{t}\left(\rho R_{i j}\right)+\partial_{x_{k}}\left(\rho U_{k} R_{i j}\right)+\rho\left(R_{i k} \partial_{x_{k}} U_{j}+R_{j k} \partial_{x_{k}} U_{i}\right)=\rho\left(R_{i j}^{L}-R_{i j}\right) / \tau_{p}^{R}
\end{aligned}
$$

Since this system is invariant under frame rotation, we consider the reference frame $(\underline{n}, \underline{\tau}): \underline{n}=\left(n_{x}, n_{y}\right), \underline{\tau}=\left(-n_{y}, n_{x}\right)$, such that $n_{x}^{2}+n_{y}^{2}=1$, for a given interface whose normal is $\underline{n}$. The transition relations are given by: $U_{n}=\underline{U} . \underline{n}, U_{\tau}=\underline{U} \cdot \underline{\tau}, R_{n n}=$ $\underline{n}^{t} \cdot \underline{\underline{R}} \cdot \underline{n}, R_{n \tau}=\underline{n^{t}} \cdot \underline{\underline{R}} \cdot \underline{\tau}=\underline{\tau^{t}} \underline{\underline{R}} \cdot \underline{n}=R_{\tau n}, R_{\tau \tau}=\underline{\tau^{t}} \cdot \underline{\underline{R}} \cdot \underline{\tau}$.

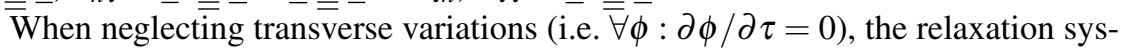
tem corresponding to system (3) for a variable $Z^{t}=\left(\rho, U_{n}, U_{\tau}, \rho R_{n n}, \rho R_{n \tau}, S\right)$ takes the form:

$$
\partial_{t} Z+A_{n}(Z) \partial_{n} Z=\mathscr{S}(Z),
$$

with $Z=Z\left(t, x_{n}\right), S=\left(\left(\rho R_{n n}\right)\left(\rho R_{\tau \tau}\right)-\left(\rho R_{n \tau}\right)^{2}\right) / \rho^{4}$ and:

$$
A_{n}(Z)=\left(\begin{array}{cccccc}
U_{n} & \rho & 0 & 0 & 0 & 0 \\
0 & U_{n} & 0 & \vartheta & 0 & 0 \\
0 & 0 & U_{n} & 0 & \vartheta & 0 \\
0 & \Psi_{n n} & 0 & U_{n} & 0 & 0 \\
0 & \Phi_{n n} & \Phi_{n \tau} & 0 & U_{n} & 0 \\
0 & 0 & 0 & 0 & 0 & U_{n}
\end{array}\right), \mathscr{S}(Z)=\left(\begin{array}{c}
0 \\
0 \\
0 \\
\rho\left(R_{n n}^{L}-R_{n n}\right) / \tau_{p}^{R} \\
\rho\left(R_{n \tau}^{L}-R_{n \tau}\right) / \tau_{p}^{R} \\
\left(S^{L}-S\right) / \tau_{p}^{R}
\end{array}\right)
$$


where $\vartheta(x, t)=1 / \rho(x, t)$. Eigenvalues of the corresponding homogeneous system:

$$
\lambda_{1,6}=U_{n} \pm c_{1}, \quad \lambda_{2,5}=U_{n} \pm c_{2}, \quad \lambda_{3}=\lambda_{4}=U_{n},
$$

with $\rho c_{1}^{2}=\Psi_{n n}$ and $\rho c_{2}^{2}=\Phi_{n \tau}$. Thus, system (4) is hyperbolic (unless vacuum occurs in the solution) if $\Psi_{n n}>0$ and $\Phi_{n \tau}>0$. Further, two distinct forms of the functions $\left(\Psi_{n n}(Z), \Phi_{n \tau}(Z), \Phi_{n n}(Z)\right)$ are introduced.

- The first one, refered to as (A1), relies on the pure Eulerian formulation and thus takes advantage of the hyperbolic structure of Eulerian closures [3]:

$$
\Psi_{n n}=3 \rho R_{n n}, \quad \Phi_{n \tau}=\rho R_{n n}, \quad \Phi_{n n}=2 \rho R_{n \tau} .
$$

In that case, system (4) is characterized by four linearly-degenerate (LD) fields and by two genuinely non-linear (GNL) fields. This approach is detailed in $[9,10]$. Its nice feature is that the whole set of partial differential equations in the evolution step preserves the realisability of the "Reynolds stress tensor" $R_{i j}$, both at the continuous and the discrete levels. This is in fact mandatory since eigenvalues remain real if and only if the quadratic form $n_{i} R_{i j} n_{j}$ remains positive. However, a drawback in this approach is due to the "true" non-conservative form of the governing equations for the Reynolds stress components in (4). Thus, non-conservative products that are active in genuinely non-linear fields are not uniquely defined.

- This has motivated the introduction of a second form for $\left(\Psi_{n n}, \Phi_{n \tau}, \Phi_{n n}\right)$ corresponding to (A2), (see [6,7] for its counterpart in the 1D framework). The main objective here is to comply with the same specifications: (i) the system should be hyperbolic, (ii) the Reynolds stress tensor should remain realisable, (iii) jump conditions should be uniquely defined, field by field. The basic idea is to introduce functions which are close enough to the first ones (6), but such that non-conservative products are only effective through linearly degenerate fields. We propose the following expression of the functions $\left(\Psi_{n n}, \Phi_{n \tau}, \Phi_{n n}\right)$ :

$$
\Psi_{n n}=3 \rho_{0}^{2}\left(R_{n n}\right)_{0} \vartheta, \quad \Phi_{n \tau}=\rho_{0}^{2}\left(R_{n n}\right)_{0} \vartheta, \quad \Phi_{n n}=2 \rho R_{n \tau} .
$$

The relaxation system corresponding to (4), (7) is indeed characterized by 6 LD fields and thus the jump relations are uniquely defined. There is only one point to be noted: system (4) with such closures no longer preserves the positivity of the Reynolds stresses neither at the continuous nor at the discrete level. Nevertheless, the possible loss of positivity does not affect the approximations of system (2), because the true values of the Reynolds stresses $R_{i j}^{L}$ are restored at the end of each time step.

From now on, we assume that the initial conditions of the Riemann problem are physically relevant:

$$
\rho_{L, R}>0, \quad \underline{x}^{t} \underline{\underline{R}}_{L, R} \underline{x}>0 .
$$


Property 1 (Existence and Uniqueness of the solution of the Riemann problem for A1). The Riemann problem associated with (4), (6), with the choice of approximate jump relations given in $[9,10]$ and with the initial conditions (8) admits a unique solution if

$$
\left(U_{n}\right)_{R}-\left(U_{n}\right)_{L}<\sqrt{3}\left(\sqrt{\left(R_{n n}\right)_{L}}+\sqrt{\left(R_{n n}\right)_{R}}\right) .
$$

The solution is composed of six constant states $Z_{L}, Z_{1}, Z_{2}, Z_{3}, Z_{4}, Z_{R}$ separated by 2 GNL waves and $4 L D$ waves associated with $\lambda_{2,3,4,5}$.

Property 2 (Existence and Uniqueness of the solution of the Riemann problem for A2). Assume that $a_{0}=3 \rho_{0}^{2}\left(R_{n n}\right)_{0} \geq 0$ is such that it satisfies the wave ordering condition (WOC): $\lambda_{1}<\lambda_{2}<\lambda_{3}=\lambda_{4}<\lambda_{5}<\lambda_{6}$. Then the Riemann problem associated with (4), (7) and initial conditions (8) admits a unique solution composed of six constant states $Z_{L}, Z_{1}^{\prime}, Z_{2}^{\prime}, Z_{3}^{\prime}, Z_{4}^{\prime}, Z_{R}$ separated by $6 L D$ waves. The (WOC) is the same as in the one-dimensional framework (see $[6,7])$.

Property 3 (Positivity of interface values of the density).

- The realisability of the solution in approach (A1) is ensured by the no vacuum occurence condition (9): $\rho_{1}=\rho_{2}>0, \rho_{3}=\rho_{4}>0$;

- For (A2), the latter condition is replaced by WOC, that guarantees the positvity of the densities in intermediate states: $\rho_{1}^{\prime}=\rho_{2}^{\prime}>0, \rho_{3}^{\prime}=\rho_{4}^{\prime}>0$.

Remark 1 (Positivity of interface values of Reynolds stresses). In the approach (A1), the positivity of the "Reynolds stresses" is required to ensure the hyperbolicity property for the corresponding relaxation system and, at the same time, is preserved by the very construction of this system, whereas in the approach (A2) the positivity of the "Reynolds stresses" in the intermediate states is not preserved for any initial condition. However, it is crucial to emphasize that at each time step the local values of $\underline{\underline{R}}^{E}=\underline{\underline{R}}^{L}$ are restored, thus the realisability is ensured owing to the instantaneous relaxation step.

\section{Stability property of both approaches A1, A2}

We focus now on the evolution step in the relaxation procedure, thus on the homogeneous system corresponding to the left hand side of (3). In order to give an estimation of the mean kinetic energy, which characterises the initial system of equations (2), we focus only on smooth solutions (we assume that: $\rho(\underline{x}, t), U_{i}(\underline{x}, t)$, $\left.R_{i j}(\underline{x}, t) \in \mathscr{C}^{1}, i, j=1,2\right)$, and we study the evolution of the "total" energy in the relaxation system (3). Let us denote by

$$
\mathscr{E}_{1}(t)=\frac{1}{2} \int_{\Omega} \rho U_{i}^{2}(\underline{x}, t) d \Omega \quad \text { and } \quad \mathscr{E}_{2}(t)=\frac{1}{2} \int_{\Omega} \rho \operatorname{tr}(\underline{\underline{R}})(\underline{x}, t) d \Omega, \quad i=1,2 .
$$


the kinetic energy of the drift (the mean motion) and the energy of the fluctuating particle motion. The total particle energy is given by $\mathscr{E}(t)=\mathscr{E}_{1}(t)+\mathscr{E}_{2}(t)$. We also assume that: $\forall \underline{x} \in \partial \Omega \quad \underline{U}_{p} \cdot \underline{n}=0$, where $\underline{U}_{p}$ is the instantaneous particle velocity.

Property 4 (Energy estimation for A1). We define: $\delta=R_{11} R_{22}-R_{12}^{2}$ and we assume that $\delta\left(\underline{x} \in \partial \Omega, t>t_{0}\right)>0, \delta\left(\underline{x} \in \Omega, t_{0}\right)>0$. Then smooth solutions of the homogeneous relaxation system corresponding to approach (A1) satisfy the following estimate:

$$
0 \leq \mathscr{E}_{1}(t)=\mathscr{E}\left(t_{0}\right)-\mathscr{E}_{2}(t) \leq \mathscr{E}\left(t_{0}\right), \quad \text { since } \quad \mathscr{E}_{2}(t) \geq 0 .
$$

An important ingredient in the proof is linked with the fact that the governing equation of $X=\delta / \rho^{2}$ reads:

$$
\partial_{t} X+(\underline{U} \cdot \nabla) X=0
$$

Up to now, we can only give a partial estimation for approach (A2). Actually, for the system corresponding to (4), (7), we must introduce a slightly modified definition of the total energy in a $1 D$ framework:

$$
\tilde{\mathscr{E}}=\mathscr{E}_{1}(t)+\mathscr{E}_{2}(t)+\int_{\Omega} \frac{\rho\left(a_{0}^{2} \vartheta-3 \rho R_{n n}\right)^{2}}{16 a_{0}^{2}} d \Omega, \quad \text { with } a_{0}^{2}=3 \rho_{0}^{2}\left(R_{n n}\right)_{0},
$$

and we only have the following estimation (see [7]):

Remark 2 (Energy estimation for A2). In a one-dimensional framework, smooth solutions of the homogeneous relaxation system corresponding to (4), (7) satisfy:

$$
0 \leq \mathscr{E}_{1}(t) \quad \text { and } \quad \mathscr{E}_{1}(t)+\mathscr{E}_{2}(t) \leq \tilde{\mathscr{E}}\left(t_{0}\right)
$$

However, no lower bound on $\mathscr{E}_{2}(t)$ has been found yet. Thus, no upper bound for $\mathscr{E}_{1}(t)$ is available yet.

\section{Numerical algorithm and results}

In order to compute the approximations of solutions of system (2) at each time step we use a fractional step method, which proceeds in three steps:

- Step 1 (Evolution): computes the approximations $\rho^{n+1,-},\left(\rho U_{i}\right)^{n+1,-},\left(\rho R_{i j}\right)^{n+1,-}$, $(i, j) \in\{1,2\}^{2}$ of the homogeneous system corresponding to the left hand side of (3) using an approximate Godunov solver for (A1) [10] and an exact Godunov solver for (A2) (using results of property 2).

- Step 2 (Relaxation): restores local values of the Reynolds stresses $R_{i j}=R_{i j}^{L}$ :

$$
\rho^{n+1}=\rho^{n+1,-}, \quad\left(\rho U_{i}\right)^{n+1}=\left(\rho U_{i}\right)^{n+1,-}, \quad\left(\rho R_{i j}\right)^{n+1,-}=\rho^{n+1}\left(R_{i j}\right)^{L} .
$$

- Step 3 (Sources): accounts for physical source terms (right hand side of (2)). 
Analytical test cases: In order to validate the two approaches (A1, A2), we consider some test cases where analytical solutions are known and we focus especially on the most difficult configurations. Assuming the following closure relation: $\rho R_{i j}^{L}=$ $S_{0} \rho^{\gamma} \delta_{i j}$ with constant entropy $S_{0}=10^{5}$ and $\gamma=3$ (this value of $\gamma$ corresponds to the isentropic case arising in $[3,10])$, we focus on two 1D Riemann problems. The computational domain is a square $[-1,1]^{2}$, the time step is in agreement with the CFL condition $(\mathrm{CFL}=0.49)$, and the regular meshes contain from $2 \times 10^{2}$ up to $2 \times 10^{5}$ cells. The figures below (fig. 1 ) represent the $L^{1}$-norm of the errors w.r.t. the mesh size. On the whole, both methods (A1, A2) guarantee the correct convergence of approximations. Moreover, we retrieve the classical $h^{1}$ convergence since no LD wave is involved here. Whereas (A1) and (A2) schemes exhibit almost the same accuracy, (A2) seems to be a little bit more stable than (A1). Both schemes can handle vacuum occurence and strong shock waves.
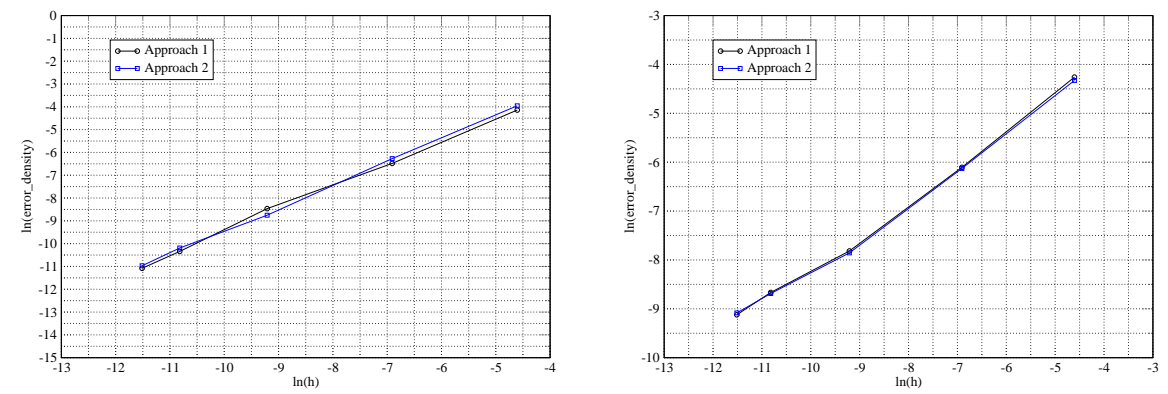

Fig. 1 L1 convergence curves for symmetric double shock (left) and symmetric double rarefaction waves with vacuum occurence (right). Coarser mesh: 200 cells; finer mesh: 200000 cells.

Numerical results with noisy Reynolds stresses: We choose the initial conditions of a subsonic shock tube problem and we plug noisy Reynolds stresses in the system of equations (2) at each time step in the cells that belong to the region $x \in[-0.25,0.25]$ as follows: $(\rho R)_{i j}^{L}=S_{0} \rho^{\gamma} \delta_{i j}(1+\operatorname{rms}(0.5-\operatorname{rand}(0,1)))$, where rms stands for the noise intensity and rand allows to manage the noise amplitude. The noisy region is not developping in time (fig. 2). The same remark holds for other values of the noise intensity. Other test cases with noisy data [6] show that the noise is independent of the mesh refinement. Moreover, the $L^{1}$ norm of the difference between approximations taking noise into account - or not - tends to be constant. Eventually, the difference between noisy approximations and those without a noise is increasing with rms in a linear manner. Both methods enable to obtain stable approximations with noisy data.

Acknowledgements The first author receives a financial support through an EDF-CIFRE contract 203/2009. Computational facilities were provided by EDF. 

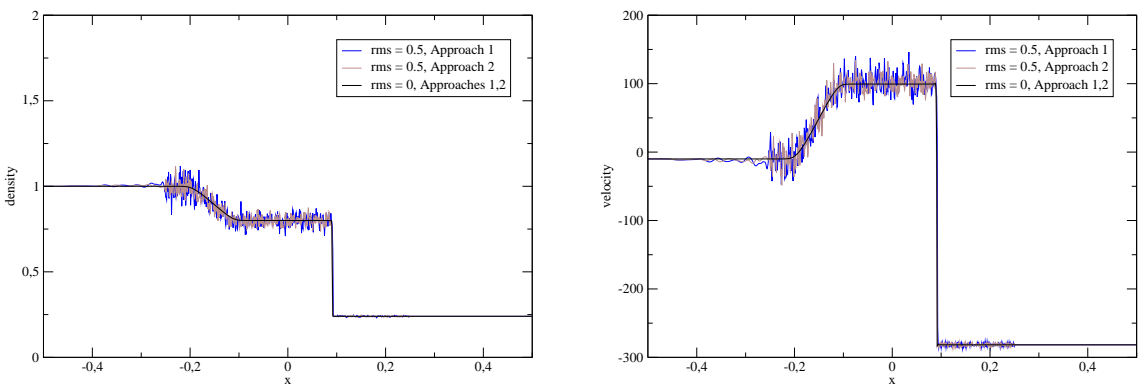

Fig. 2 Approximations of the density (left) and the velocity (right) with $\mathrm{rms}=0.5$ and $\mathrm{rms}=0$ in time. Mesh size: 1000 cells

\section{References}

1. Ambroso, A., Chalons, C., Coquel, F., Galié, T.: Relaxation and numerical approximation of a two-fluid two-pressure diphasic model. ESAIM: M2AN, 43 (6), 1063-1097 (2009)

2. Audebert, B.: Contribution à l'analyse des modèles aux tensions de Reynolds pour l'interaction choc-turbulence. Université Pierre et Marie Curie, $\mathrm{PhD}$ thesis (2006)

3. Berthon, C., Coquel, F., Hérard, J.M., Uhlmann, M.: An approximate solution of the Riemann problem for a realisable second-moment turbulent closure. Shock Waves Journal, 11 (4), 245269 (2002)

4. Bouchut, F.: Nonlinear stability of finite volume methods for hyperbolic conservation laws and well-balanced schemes for source. Frontiers in Mathematics Series, Birkhauser (2004)

5. Chibbaro, S., Hérard, J.M., Minier, J.P.: A novel Hybrid Moments/Moments-PDF method for turbulent two-phase flows. Final Technical Report Activity Marie Curie Project. TOK project LANGE Contract MTKD-CT-2004 509849 (2006)

6. Dorogan, K., Hérard, J.M., Minier, J.P.: Development of a new scheme for hybrid modelling of gas-particle two-phase flows. EDF report H-I81-2010-2352-EN, unpublished, 1-50 (2010)

7. Dorogan, K., Hérard, J.M., Minier, J.P.: A relaxation scheme for hybrid modelling of gasparticle flows. Submitted (2011)

8. Hérard, J.M.: A relaxation tool to compute hybrid Euler-Lagrange compressible models. AIAA paper 2006-2872 (2006) http://aiaa.org

9. Hérard, J.M., Minier, J.P., Chibbaro, S.: A Finite Volume scheme for hybrid turbulent twophase flow models. AIAA paper 2007-4587, http://aiaa.org

10. Hérard, J.M., Uhlmann, M., Van der Velden, D.: Numerical techniques for solving hybrid Eulerian Lagrangian models for particulate flows. EDF report H-I81-2009-3961-EN, unpublished (2009)

11. Jin, S., Xin, Z.: The relaxation schemes for systems of conservation laws in arbitrary space dimensions. Comm. Pure Appl. Math., 48, 235-276 (1995)

12. Minier, J.P., Peirano, E.: The pdf approach to polydispersed turbulent two-phase flows. Physics reports, 352, 1-214 (2001)

13. Muradoglu, M., Jenny, P., Pope, S.B., Caughey, D.A.: A consistent hybrid finite-volume/ particle method for the pdf equations of turbulent reactive flows. J. Comp. Phys., 154, 342371 (1999)

14. Peirano, E., Chibbaro, S., Pozorski, J., Minier, J.P.: Mean-field/PDF numerical approach for polydispersed turbulent two-phase flows. Prog. Ene. Comb. Sci., 32, 315-371 (2006) 